\title{
Rheo-Optical Studies on the Deformation Mechanism of Semicrystalline Polymers. V. Quantitative Analysis of Grain-Boundary Relaxation in Polyethylene
}

\author{
Shoji SueHIRo, Thein KyU, Ken-ichi FujITa, and Hiromichi KawaI \\ Department of Polymer Chemistry, Faculty of Engineering, \\ Kyoto University, Kyoto 606, Japan.
}

(Received August 25, 1978)

\begin{abstract}
The dynamic mechanical behavior of a low-density polyethylene above room temperature was analyzed in terms of the $\alpha$ and $\beta$ mechanical dispersions, using a mechanical model reflecting its spherulitic crystalline texture. The mechanical $\alpha$ dispersion, assigned to a crystal grain-boundary relaxation within the crystal lamellae, is quantified in terms of the dynamic orientation of the crystal grains on the basis that the dynamic orientation is proportional to fractional elongation of the grain-boundary region. The mechanical $\beta$ dispersion, though only a part of its entire spectrum, is also quantified by constructing its master curve; the activation energy of the relaxation process is found to be around $27 \mathrm{kcal} /$ $\mathrm{mol}\left(113 \mathrm{kJmol}^{-1}\right)$. The significance of the vertical shift factors for dynamic mechanical and optical data is also discussed in terms of temperature dependences of the structural and mechanical parameters of the model. It is found that the mechanical shift factor is related to both the temperature dependences of the crystal elasticity as well as to its crystallinity, especially, to the former, whereas the optical shift factor is mostly related to the latter.

KEY WORDS Rheo-Optics / Polyethylene / Mechanical $\alpha_{1}$ Dispersion / Grain-Boundary Relaxation / Spherulite Deformation Model / Vertical Shift Factor /
\end{abstract}

The mechanical $\alpha$ dispersion of polyethylene, especially the $\alpha_{1}$ dispersion, has been studied by Iwayanagi, et al., ${ }^{1,2}$ McCrum and Morris, ${ }^{3}$ and Takayanagi, et al., ${ }^{4-6}$ in terms of mechanical and morphological models to assign the dispersion to a sort of grain-boundary phenomena. Their approaches were, however, not necessarily adequate for deducing direct evidence for this assignment, and were not sufficiently quantitative in evaluating the grain-boundary dispersion.

It is well-known that the semicrystalline polymers including polyethylene are the thermo-rheologically complex materials requiring not only the horizontal shift(s) but also an unexpected amount of vertical shift of the mechanical data at various temperatures, such as the dynamic modulus and relaxation modulus functions, for constructing the so-called master curves of the functions corresponding to the mechanical $\alpha_{1}$ dispersion. The significance of the vertical shift was discussed by Nagamatsu, et al., ${ }^{7}$ and Onogi, et al. ${ }^{8}$ in terms of temperature dependence on the degree of crystallinity and the NMR mobile fraction in the material. These problems are not yet completely solved. Kajiyama, et al., ${ }^{4}$ also discussed the relaxation strength of the mechanical $\alpha_{1}$ dispersion in terms of volume fraction of the intermosaic block region (grain-boundary region) in the material and its effective dynamic viscosity, semiquantitatively. But the problem of identifying the vertical shift is still unsolved.

In a previous paper ${ }^{9}$ of this series of rheooptical studies on the mechanical $\alpha$ dispersion of spherulitic films of a low-density polyethylene, the following three conclusions were reached, i.e., (1) crystal lattice deformation is in-phase with respect to the applied stress, suggesting the polymer crystal to be very likely elastic in the 
dynamic response, (2) noncrystalline orientation is also in-phase with respect to the applied strain, and, (3) crystal orientation is, in contrast to (2), much lagged behind the applied strain. Also it was revealed that the dynamic crystal orientation shows a definite frequency dispersion in the same frequency range as the mechanical $\alpha$ dispersion of the material, when the data for both were reduced to a common reference temperature, with an activation energy of orientation relaxation process of around $22 \mathrm{kcal} / \mathrm{mol}$. The value of the activation energy was found to be consistent with the activation energy of mechanical relaxation process of the $\alpha_{1}$ dispersion. Assuming that the crystal orientation relaxation arose from a grainboundary phenomenon, the above three significant results from (1) through (3) are explainable qualitatively in terms of a simple mechanical model of spherulite deformation.

The unsolved problems are then: how to relate the mechanical $\alpha_{1}$ dispersion to the dynamic crystal orientation dispersion in a more quantitative way by taking into account the grain-boundary effect and how to pursue the origin of the vertical shift of dynamic mechanical data for constructing the master curves. The qualitative model of spherulite deformation used in the previous paper will be modified to some extent, taking into account fractional volumes of crystalline and noncrystalline materials as well as the grainboundary material so as to quantify the discussion about the grain-boundary relaxation and to identify the vertical shift of mechanical and optical (birefringence) data.

\section{MECHANICAL MODEL OF LOW- DENSITY POLYETHYLENE}

The polyethylene spherulite is composed of crystal lamellae which have grown radially with the crystal $b$ axis parallel to the growth direction around which the lamella is twisted, thus rotating the crystal $a$ axis and $c$ axis periodically. Noncrystalline chain-molecules are believed to exist in the interlamellar region(s) as tie-chains, loops, cilia, and free chains. When the spherulite is deformed uniaxially, there may be two extreme cases in which the crystalline and noncrystalline phases are coupled in parallel and series fashions, respectively, against the tensile stress, as demon-

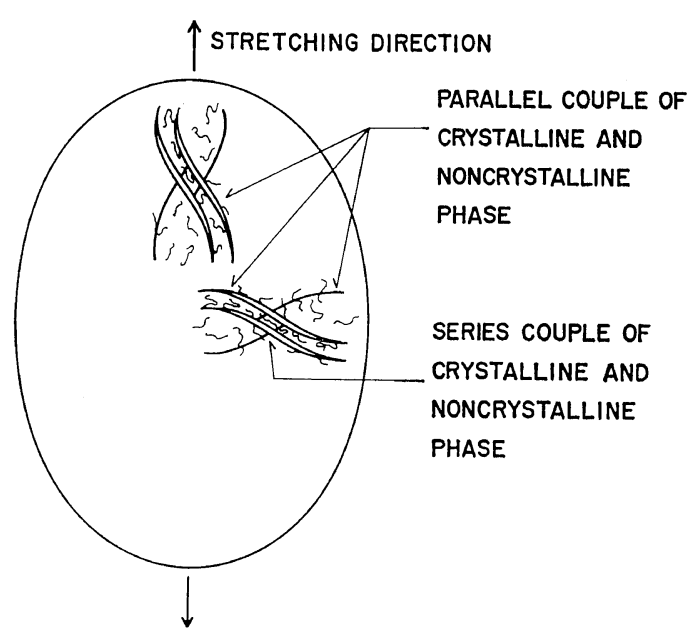

Figure 1. Schematic illustration of an uniaxial deformation of polyethylene spherulite.

strated in Figure 1. In the polar zone of the deformed spherulite, for example, the two phases may be coupled in parallel fashion, while in the equatorial zone, the two phases may be coupled in parallel or series fashion, depending on the twisting growth of the lamellae.

Although the model of spherulite deformation in Figure 1 is an extreme one, not taking into account any shear deformation of the phases, the model may be schematized by a simple mechanical model, as given in Figure 2. The mechanical model is based on a homogeneous strain hypothesis between the $\mathrm{P}$ and $\mathrm{S}$ components, where $\mathrm{P}$ and $\mathrm{S}$ denote parallel and series couples of the two phases, respectively, and $\phi$ denotes the volume fraction of the $\mathbf{P}$ component with possibly $\phi>$ $(1-\phi)$. Similar to a proposal by Kajiyama, et al., ${ }^{4}$ the $\mathrm{P}$ and $\mathrm{S}$ components in Figure 2 may be further modified, as shown in Figures 3(a) and

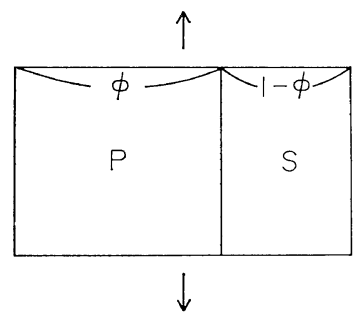

Figure 2. Equivalent mechanical model for the uniaxial deformation of spherulite in Figure 1. 
P-component

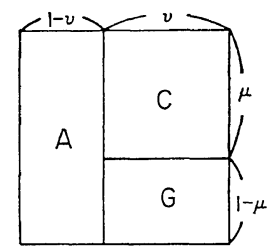

(a)
S-component

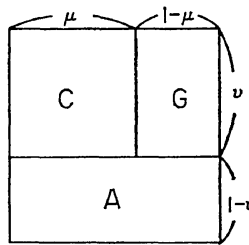

(b)
Figure 3. Parallel couple component (a) and series couple component (b) constituting the mechanical model in Figure 2.

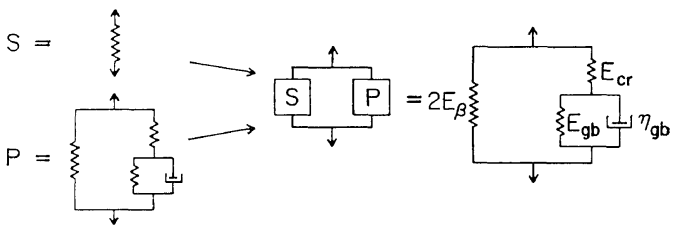

Figure 4. A qualitative model to account for mechanical properties of a low-density polyethylene above $T_{g}$, proposed in a previous paper.

3(b) respectively, by considering that the crystal grain-boundary arises from the paracrystalline nature of the crystal lamellae, as fully discussed in the previous paper. ${ }^{9}$ In Figure 3, C, A, and G denote the crystalline (lamella), noncrystalline (interlamellar), and crystal grain-boundary phases, respectively, with volume fractions of $\nu \mu,(1-\nu)$, and $\nu(1-\mu)$, respectively.

In Figure 4, a mechanical model proposed in the previous paper is again illustrated for comparison, where neither the volume fraction of $\phi$, $\nu$ nor $\mu$ is taken into account, and the $\mathrm{S}$ component is approximated only by $\mathrm{A}$ because of its much greater elastic compliance than $\mathrm{C}$ plus $\mathrm{G}$ in parallel. It is, however, obvious that the mechanical model in Figure 4 is essentially the same as that in Figure 2, at least qualitatively.

\section{Mechanical Properties}

Let us investigate the mechanical properties of the model in Figure 2, such as the complex dynamic modulus function, $E^{*}$. In the following calculations, the mechanical response is always accounted against sinusoidal excitation, so that modulus, strain and stress are expressed in terms of complex quantities.

First, the complex dynamic modulus of the $\mathrm{P}$ component in Figure 3(a) may be given by

$$
E_{\mathrm{P}}^{*}=\nu E_{\mathrm{cr}, \mathrm{P}}^{*}\left(\gamma_{\mathrm{er}, \mathrm{P}}^{*} / \gamma^{*}\right)+(1-\nu) E_{\mathrm{am}}^{*}
$$

where $E$ and $\gamma$ denote tensile modulus and strain, and subscripts $P$, cr, and am denote the $P$ component, the crystalline phase, and the noncrystalline phase, respectively. In the $\mathbf{P}$ component, the direction of the molecular chains in the crystalline phase is perpendicular to the stretching direction, and so $E_{\mathrm{cr}, \mathrm{P}}^{*}$ may correspond to the mean crystal modulus in the direction perpendicular to the molecular axis. The complex dynamic strain of the crystalline phase, $\gamma_{\mathrm{cr}, \mathrm{P}}^{*}$ may be given by

$$
\gamma_{\mathrm{cr}, \mathrm{P}}^{*}=\Delta l_{\mathrm{cr}, \mathrm{P}}^{*} / \mu l=\left(\Delta l^{*}-\Delta l_{\mathrm{gb}, \mathrm{P}}^{*}\right) \mu l
$$

where $\Delta l^{*}$ is the complex dynamic amplitude of elongation and subscript $\mathrm{gb}$ denotes the grainboundary phase. Combining eq 2 with eq 1 , it follows that

$$
E_{\mathrm{P}}^{*}=\left(\nu E_{\mathrm{cr}, \mathrm{P}}^{*} / \mu\right)\left[1-\left(\Delta l_{\mathrm{gb}, \mathrm{P}}^{*} / \Delta l *\right)\right]+(1-\nu) E_{\mathrm{am}}^{*}
$$

The ratio of $\Delta l_{\mathrm{g}, \mathrm{P}}^{*} / \Delta l^{*}$ is assumed to be proportional to the dynamic orientation of the crystal grains which may, as a first approximation, be represented by $C_{j}{ }^{*}$, the complex dynamic strainorientation coefficient of the reciprocal lattice vector of the $j$-th crystal plane,

$$
\Delta l_{\mathrm{gb}, \mathrm{p}}^{*} / \Delta l^{*}=k_{j} C_{j}^{*}
$$

where $k_{j}$ is the proportional constant.

For polyethylene, the crystal $c$ axis may be the most appropriate one for describing the orientation of the crystal grains. Finally, $E_{\mathrm{P}}{ }^{*}$ can be written by

$$
E_{\mathrm{P}}^{*}=\left(\nu E_{\mathrm{cr}, \mathrm{p}}^{*} / \mu\right)\left(1-k_{j} C_{j}^{*}\right)+(1-\nu) E_{\mathrm{am}}^{*}
$$

The complex dynamic modulus of the $\mathrm{S}$ component in Figure 3(b) may be given by

$$
\frac{1}{E_{\mathrm{s}}^{*}}=\frac{1-\nu}{E_{\mathrm{am}}^{*}}+\frac{\nu}{\mu E_{\mathrm{cr}, \mathrm{s}}^{*}+(1-\mu) E_{\mathrm{gb}}^{*}}
$$

In the $\mathrm{S}$ component, the direction of molecular chains in the crystalline phase is parallel to the stretching direction, and $E_{\mathrm{cr}, \mathrm{s}}^{*}$ corresponds to the crystal modulus in the direction of molecular axis. Since $\left|E_{\mathrm{cr}, \mathrm{s}}^{*}\right|$ is much greater than $\left|E_{\mathrm{am}}^{*}\right|$, the strain of the crystalline phase and the grainboundary phase is negligibly small, compared to that of the noncrystalline phase; thus eq 6 can be reduced to

$$
E_{\mathrm{s}}^{*} \simeq E_{\mathrm{am}}^{*} /(1-\nu)
$$

From eq 5 and 7 , the complex dynamic modulus 
function of the mechanical model in Figure 2 can be deduced as

$$
E^{*}=\frac{\phi \nu E_{\mathrm{cr}, \mathrm{P}}^{*}}{\mu}\left(1-k_{j} C_{j}^{*}\right)+\left[\frac{1-\phi}{1-\nu}+\phi(1-\nu)\right] E_{\mathrm{am}}^{*}
$$

$C_{j}{ }^{*}$ are measurable quantities from the dynamic $\mathrm{X}$-ray diffraction, as fully discussed in the previous papers, ${ }^{9,10}$ and eq 8 quantifies the elastic modulus of spherulite $E^{*}$ in terms of $C_{j}{ }^{*}$ within a crude approximation of mechanical model of spherulite in Figures 1 through 3.

\section{Optical Properties}

Denoting complex dynamic amplitudes of birefringence in the crystalline, noncrystalline, and grain-boundary phases in the $\mathrm{P}$ component as $\Delta_{\mathrm{cr}, \mathrm{p}}^{*}, \Delta_{\mathrm{am}, \mathrm{P}}^{*}$, and $\Delta_{\mathrm{gb}, \mathrm{P}}^{*}$, respectively, these can be written as

$$
\begin{aligned}
& \Delta_{\mathrm{cr}, \mathrm{P}}^{*}=K_{\mathrm{cr}, \mathrm{P}}^{\mathrm{ori}} \gamma^{*}+K_{\mathrm{cr}, \mathrm{P}}^{\mathrm{dis} *} \gamma_{\mathrm{cr}, \mathrm{P}}^{*} \\
& \Delta_{\mathrm{am}, \mathrm{P}}^{*}=K_{\mathrm{am}, \mathrm{P}}^{*} \gamma_{\mathrm{am}, \mathrm{P}}^{*}=K_{\mathrm{am}, \mathrm{p}}^{*} \gamma^{*} \\
& \Delta_{\mathrm{gb}, \mathrm{P}}^{*}=K_{\mathrm{gb}, \mathrm{p}}^{*} \gamma_{\mathrm{gb}, \mathrm{P}}^{*}
\end{aligned}
$$

where $K$ is the strain-optical coefficient. The first term on the right-hand side of eq 9 contributes to the crystalline birefringence due to crystal orientation, which is written as

$$
K_{\mathrm{cr}, \mathrm{p}}^{\mathrm{or} 1}=\left(n_{\mathrm{a}}-n_{\mathrm{c}}\right) C_{\mathrm{a}}{ }^{*}+\left(n_{\mathrm{b}}-n_{\mathrm{c}}\right) C_{\mathrm{b}}{ }^{*}
$$

where $\left(n_{\mathrm{a}}-n_{\mathrm{c}}\right)$ and $\left(n_{\mathrm{b}}-n_{\mathrm{c}}\right)$ are the intrinsic birefringences of the crystalline phase, and $C_{\mathrm{a}}{ }^{*}$ and $C_{\mathrm{b}}{ }^{*}$ are the dynamic orientation coefficients of the crystal $a$ axis and $b$ axis. The second term on the right-hand side of eq 9 also contributes to the crystalline birefringence owing to crystal distortion (deformation), and may be much smaller than the first term above $T_{\mathrm{g}}$.

Now, it seems that the strain-optical coefficient of the crystalline phase should be discussed. In principle, the strain-optical coefficient of a given $x$-th phase must be defined by $\left(\partial \Delta_{x} / \partial \gamma_{x}\right)$. Therefore, $K_{\mathrm{cr}}^{\mathrm{or}} *$ is uncertain in its physical meaning because of crystal orientation being not simply related to the strain of the crystalline phase itself, while $K_{\mathrm{cr}}^{\mathrm{dist*}}$ and $K_{\mathrm{am}}^{*}$ are clear in their physical meaning. Actually, as discussed previously, the crystal orientation has been related to the elongation of the grain-boundary phase rather than to the crystal strain, and the first term on right- hand side of eq 9, i.e., $\Delta_{\mathrm{cr}, \mathrm{P}}^{\mathrm{or}} *$ can be formulated in terms of $\gamma_{\mathrm{B}, \mathrm{P}}^{*}$ as follows;

$$
\Delta_{\mathrm{cr}, \mathrm{P}}^{\mathrm{orr}}=\left[\left(n_{\mathrm{a}}-n_{\mathrm{c}}\right) C_{\mathrm{a}}{ }^{*}+\left(n_{\mathrm{b}}-n_{\mathrm{c}}\right) C_{\mathrm{b}}{ }^{*}\right] r^{*}
$$

Since $C_{\mathrm{a}}{ }^{*}$ and $C_{\mathrm{b}}{ }^{*}$ can be given by

$$
\begin{aligned}
& C_{\mathrm{a}}{ }^{*}=k_{\mathrm{a}}\left(\Delta l_{\mathrm{g}} / \Delta l^{*}\right)=k_{\mathrm{a}}(1-\mu)\left(\gamma_{\mathrm{g} b}^{*} / \gamma^{*}\right) \\
& C_{\mathrm{b}}{ }^{*}=k_{\mathrm{b}}(1-\mu)\left(\gamma_{\mathrm{g}}^{*} / \gamma^{*}\right)
\end{aligned}
$$

Equation 13 becomes

$$
\Delta_{\mathrm{cr}, \mathrm{P}}^{\mathrm{ori}} *=\left[k_{\mathrm{a}}\left(n_{\mathrm{a}}-n_{\mathrm{c}}\right)+k_{\mathrm{b}}\left(n_{\mathrm{b}}-n_{\mathrm{c}}\right)\right](1-\mu) \gamma_{\mathrm{g} b}^{*}
$$

For actual calculation of $\Delta_{\mathrm{cr}, \mathrm{p}}^{\mathrm{or}} *$, however, its representation by eq 13 , rather than eq 14 , may be used since $C_{\mathrm{a}}{ }^{*}$ and $C_{\mathrm{b}}{ }^{*}$ are measurable quantities of the crystal orientation response. In other words, $K_{\mathrm{cr}, \mathrm{P}}^{\mathrm{ori}} *$ adopted here is not a conventional one as defined by $\left(\partial \Delta_{\mathrm{cr}} / \partial \gamma_{\mathrm{cr}}\right)$ but as $\left(\partial \Delta_{\mathrm{cr}}^{\mathrm{or}} / \partial \gamma\right)$; i.e., a ratio of crystal birefringence increment arising from crystal orientation against the applied strain.

Assuming the second term of eq 9 owing to the crystal distortion and $\Delta_{\mathrm{gb}, \mathrm{P}}^{*}$ to be negligibly small, the dynamic birefringence of the $\mathbf{P}$ component $\Delta_{\mathbf{P}}{ }^{*}$ may be given by

$$
\begin{aligned}
\Delta_{\mathrm{P}}^{*}=\nu \mu\left[\left(n_{\mathrm{a}}-n_{\mathrm{c}}\right) C_{\mathrm{a}}{ }^{*}\right. & \left.+\left(n_{\mathrm{b}}-n_{\mathrm{c}}\right) C_{\mathrm{b}}{ }^{*}\right] \gamma^{*} \\
& +(1-\nu) K_{\mathrm{am}, \mathrm{p}}^{*} \gamma^{*}
\end{aligned}
$$

Similar to eq 9 through 11, the dynamic amplitudes of birefringence in the $\mathrm{S}$ component may be given as

$$
\begin{aligned}
& \Delta_{\mathrm{cr}, \mathrm{s}}^{*}=K_{\mathrm{cr}, \mathrm{s}}^{\mathrm{ori}} \gamma^{*}+K_{\mathrm{cr}, \mathrm{s}}^{\mathrm{dist} *} \gamma_{\mathrm{cr}, \mathrm{s}}^{*} \\
& \Delta_{\mathrm{am}, \mathrm{s}}^{*}=K_{\mathrm{am}, \mathrm{s}}^{*} \gamma_{\mathrm{am}, \mathrm{s}}^{*} \\
& \Delta_{\mathrm{g}, \mathrm{s}}^{*}=K_{\mathrm{g} b, \mathrm{~s}}^{*} \gamma_{\mathrm{g}, \mathrm{s}}^{*}
\end{aligned}
$$

Since $\left|E_{\mathrm{cr}, \mathrm{s}}^{*}\right| \gg\left|E_{\mathrm{am}, \mathrm{s}}^{*}\right|$, both $\left|\gamma_{\mathrm{cr}, \mathrm{s}}^{*}\right|$ and $\left|\gamma_{\mathrm{g}, \mathrm{s}}^{*}\right|$ are much smaller than $\left|\gamma_{\mathrm{am}, \mathrm{s}}^{*}\right|$. This makes the contributions of $\Delta_{\mathrm{cr}, \mathrm{s}}^{*}$ and $\Delta_{\mathrm{s}, \mathrm{s}}^{*}$ to $\Delta_{\mathrm{s}}{ }^{*}$ negligible, and $\Delta_{\mathrm{s}} *$ can be written as

$$
\Delta_{\mathrm{s}}^{*} \simeq(1-\nu) K_{\mathrm{am}, \mathrm{s}}^{*} \gamma_{\mathrm{am}}^{*} \simeq K_{\mathrm{am}, \mathrm{s}}^{*} \gamma^{*}
$$

Overall birefringence $\Delta^{*}$ may be given by

$$
\begin{aligned}
\Delta^{*}= & \phi \Delta_{\mathrm{P}}{ }^{*}+(1-\phi) \Delta_{\mathrm{s}}{ }^{*} \\
= & \phi \nu \mu\left[\left(n_{\mathrm{a}}-n_{\mathrm{c}}\right) C_{\mathrm{a}}{ }^{*}+\left(n_{\mathrm{b}}-n_{\mathrm{c}}\right) C_{\mathrm{b}}{ }^{*}\right] \gamma^{*} \\
& +\phi(1-\nu) K_{\mathrm{am}, \mathrm{P}}^{*} \gamma^{*}+(1-\phi) K_{\mathrm{am}, \mathrm{s}}^{*} \gamma^{*}
\end{aligned}
$$

Assuming $K_{\mathrm{am}, \mathrm{P}}^{*}=K_{\mathrm{am}, \mathrm{s}}^{*} \equiv K_{\mathrm{am}}^{*}$, though $K_{\mathrm{am}, \mathrm{P}}^{*}$ is not rigorously identical to $K_{\mathrm{am}, \mathrm{s}}^{*}$, the overall strain-optical coefficient $K^{*}$ may be given by 


$$
\begin{aligned}
K^{*}=\Delta^{*} / \gamma^{*} \simeq \phi \nu \mu\left[\left(n_{\mathrm{a}}-n_{\mathrm{c}}\right) C_{\mathrm{a}}{ }^{*}\right. & \left.+\left(n_{\mathrm{b}}-n_{\mathrm{c}}\right) C_{\mathrm{b}}{ }^{*}\right] \\
& +(1-\phi \nu) K_{\mathrm{am}}^{*}
\end{aligned}
$$

Takayanagi et al., ${ }^{11}$ discussed the strain-optical coefficient of semicrystalline polymers, two-phase system of crystalline and noncrystalline phases, in terms of an equivalent model method, and denied the conventional expression given by

$$
K^{*}=K_{\mathrm{cr}}^{*}\left(1-X_{\mathrm{am}}\right)+K_{\mathrm{am}}^{*} X_{\mathrm{am}}
$$

They suggested that the fractional volume of noncrystalline phase $X_{\text {am }}$ must be replaced with a dimensionless complex number $R^{*}$ which is related not only to $X_{\mathrm{am}}$ but also to $E_{\mathrm{cr}}^{*}$ and $E_{\mathrm{am}}^{*}$ as well. Their discussion seems to be rational, if the simple two-phase model with homogenious strain hypothesis is not valid. The essential problem arises, however, from the obscurity in the physical meaning of $K_{\mathrm{cr}}^{*}$, as discussed above, and either eq 12 or 13 must be adopted for representing crystalline birefringence, unless the birefringence results from crystal distortion.

\section{RESULTS AND DISCUSSION}

\section{Separation of $\alpha$ and $\beta$ Dispersions from $E^{*}$}

Let us discuss the separation of $\alpha$ (grainboundary) and $\beta$ (primary) dispersions from $E^{*}$ by utilizing the dynamic mechanical and crystal orientation data obtained in a previous paper ${ }^{9}$ for a spherulitic film of polyethylene, a meltcrystallized and annealed film of a low-density polyethylene (Sumikathene G201). As discussed in the previous paper, the polymer crystal is very likely to be elastic in its mechanical response, leading $E_{\mathrm{cr}}^{*}=E_{\mathrm{cr}}$ and, further, reforming eq 8 as

$$
E^{*}=p\left(1-k_{j} C_{j}^{*}\right)+q E_{\mathrm{am}}^{*}
$$

where

$$
p=\phi \nu E_{\mathrm{cr}} / \mu
$$

and

$$
q=\frac{1-\phi}{1-\nu}+(1-\nu)
$$

The first and second terms on the right-hand side of eq 23 can be understood as contributions from the grain-boundary dispersion and the primary dispersion, respectively. The first term can be evaluated by adopting one of the measurable quantities of $C_{j}{ }^{*}$, for example $C_{\mathrm{c}}{ }^{*}$ for the crystal $c$ axis, and the proper values of $k_{\mathrm{c}}$ and $p$, and therefore, the separation can be performed by subtracting the first term thus evaluated, from $E^{*}$. That is;

$$
\begin{gathered}
E^{\prime}=E_{\mathrm{B}}{ }^{\prime}+q E_{\mathrm{am}}^{\prime} \\
E^{\prime \prime}=E_{\mathbf{B}}{ }^{\prime \prime}+q E_{\mathrm{am}}^{\prime \prime}
\end{gathered}
$$

where $E_{\mathrm{B}}{ }^{\prime}$ and $E_{\mathrm{B}}{ }^{\prime \prime}$ are real and imaginary components of the grain-boundary contribution and are rewritten by

$$
\begin{gathered}
{E_{\mathrm{B}}}^{\prime}=p\left(1-k_{\mathrm{c}} C_{\mathrm{c}}{ }^{\prime}\right) \\
{E_{\mathrm{B}}}^{\prime \prime}=-p k_{\mathrm{c}} C_{\mathrm{c}}{ }^{\prime \prime}
\end{gathered}
$$

In the upper and lower halves of Figure 5 shown are the frequency dependence of $E^{\prime}$ and $E^{\prime \prime}$ for the annealed low-density polyethylene, G201(A), respectively, both at various temperatures from 30 to $80^{\circ} \mathrm{C}$. In upper and lower halves of Figure 6 are also shown the frequency dependences of $C_{\mathrm{c}}{ }^{\prime}$ and $C_{\mathrm{c}}{ }^{\prime \prime}$ for the same specimen at the same temperatures, obtained from dynamic X-ray diffraction measurements. ${ }^{9,10}$

As has been fully discussed in the previous papers, ${ }^{9,12}$ the frequency dependence of $E^{\prime \prime}$ at various temperatures in Figure 5 reveals not only the appearance of mechanical $\alpha$ dispersion within
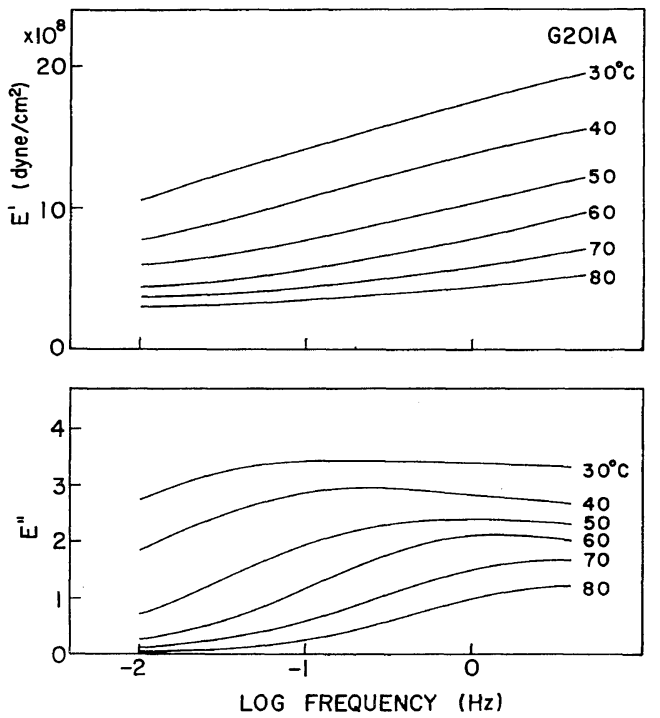

Figure 5. Temperature dependence of the storage and loss modulus functions, $E^{\prime}$ and $E^{\prime \prime}$, for an annealed film of a low-density polyethylene G201 (A). 
these ranges of frequency and temperature, but also a certain contribution of the mechanical $\beta$ dispersion at a low temperature and high frequency. It is also revealed that the mechanical dispersion,
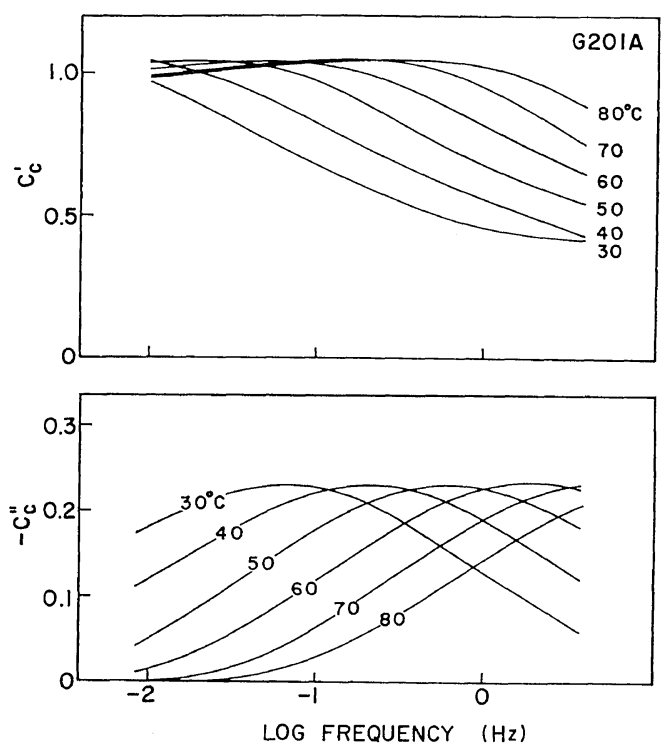

Figure 6. Temperature dependence of in-phase and out-of-phase components of the complex dynamic strain-orientation coefficient of the crystal $c$ axis, $C_{\mathrm{c}}{ }^{\prime}$ and $C_{\mathrm{c}}{ }^{\prime \prime}$, for the G201 (A) specimen.
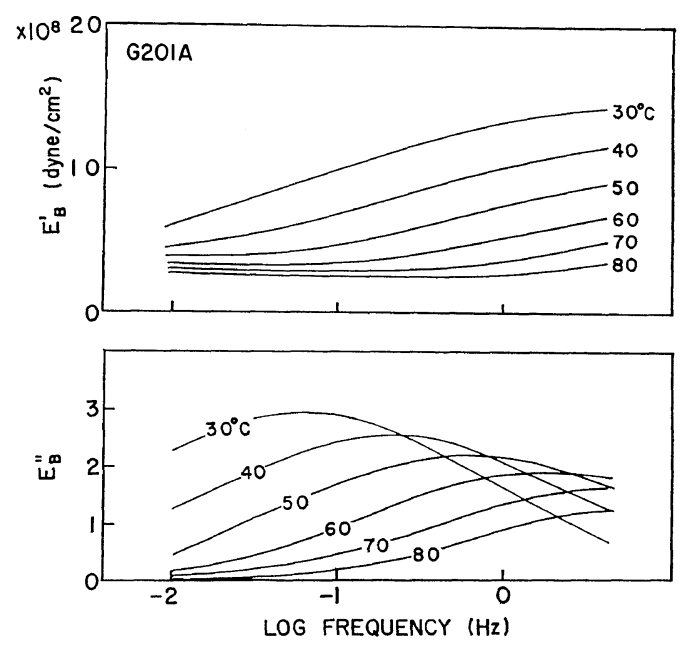

Figure 7. Temperature dependence of the storage and loss modulus functions for grain-boundary relaxation ( $\alpha_{1}$ dispersion), $E_{\mathrm{B}}{ }^{\prime}$ and $E_{\mathrm{B}}{ }^{\prime \prime}$, calculated from $C_{\mathrm{c}}{ }^{\prime}$ and $C_{\mathrm{c}}{ }^{\prime \prime}$ in Figure 6. as observed $E_{\max }^{\prime \prime}$ in Figure 5, becomes smaller as the temperature increases. This requires a temperature dependence of $p$ in eq 29 , and the dependence is assumed to be in a similar fashion as proposed by Miki, et al., ${ }^{13,14}$ for the vertical shift factor of mechanical data for semicrystalline polymers, i.e.,

$$
p=p_{0} \exp \left[-b_{0}\left(T-T_{0}\right)\right]
$$

where $T_{0}$ is a reference temperature taken as $50^{\circ} \mathrm{C}$.

Here, one has three unknown parameters, $p_{0}$, $b_{0}$, and $k_{\mathrm{c}}$, for calculating $E_{\mathrm{B}}{ }^{\prime}$ and $E_{\mathrm{B}}{ }^{\prime \prime}$ from eq 28 and 29. The parameters are determined by a trial and error method as $1.35 \times 10^{9} \mathrm{dyn} / \mathrm{cm}^{2}$ $\left(1.35 \times 10^{8} \mathrm{~Pa}\right), 0.015 \mathrm{~K}^{-1}$, and 0.68 , respectively, to give the most reasonable separation of $E^{*}$ into $E_{\mathrm{B}}{ }^{*}$ and $q E_{\mathrm{am}}^{*}$, as illustrated in Figures 7 and 8. As can be seen in the figures, $E_{\mathrm{B}}{ }^{*}$ is much greater than $q E_{\mathrm{am}}^{*}$ in its contribution to $E^{*}$, suggesting the mechanical dispersion observed here to be associated mainly with the grainboundary relaxation.
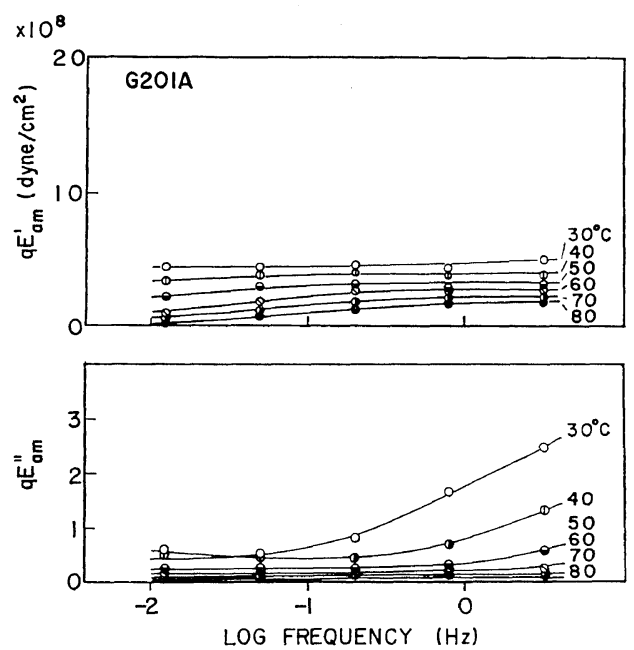

Figure 8. Temperature dependence of the storage and loss modulus functions for $\beta$ dispersion, $q E_{\mathrm{am}}^{\prime}$ and $q E_{\mathrm{am}}^{\prime \prime}$, separated from $E^{\prime}$ and $E^{\prime \prime}$.

\section{Significance of Vertical Shift Factor for Mechanical Data}

In a previous paper of this series, ${ }^{12}$ the significance of the vertical shift factor for constructing the so-called master curves of $E^{\prime}$ and $E^{\prime \prime}$ was discussed in terms of Nagamatsu's ${ }^{7}$ and Miki's ${ }^{13,14}$ 
approaches. The former approach is based on the temperature dependence of noncrystalline entropic elasticity supplemented by the volume effect of the noncrystalline regions, while the latter approach takes into account the temperature dependence of crystal elasticity based on a theory of smearingout effect on the intermolecular potential within crystal lattice proposed by Wada ${ }^{15}$ and Okano. ${ }^{16}$ Both approaches, however, give a much smaller temperature dependence of the vertical shift factor than that required for fitting the experimental data.

According to the mechanical model proposed in this paper, the significance of the vertical shift factor can be attributed to the temperature dependence of $p$ in eq 23. In other words, it must be attributed to the temperature dependence of $\nu$ and $E_{\mathrm{cr}}$ rather than to $\phi$ and $\mu$ in eq 24, as $\phi$ seems to be almost independent of the temperature and since $(1-\mu)$ must be very small in its value even though it may be temperature dependent.

Figure 9 shows the temperature dependence of $p$, calculated from eq 30 by using the estimated values of the parameter $b_{0}$, the fractional volume of crystalline phase and the dynamic crystal lattice modulus for (110) crystal plane, $X_{\mathrm{c}}$ and $E_{110}^{\prime}$, both reported in a previous paper. ${ }^{9}$ These dependences are plotted in logarithms of relative values referred to $50^{\circ} \mathrm{C}$. As seen in the figure, the temperature dependence of $E_{110}^{\prime}$ is much greater than that of $X_{\mathrm{c}}$, and the sum of their logarithmic

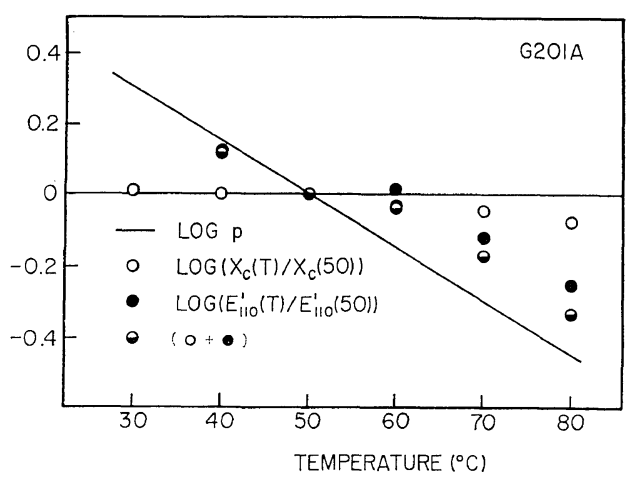

Figure 9. Temperature dependence of $p$ of eq 30 , the volume fraction of crystalline phase $X_{\mathrm{c}}$, and the dynamic crystal lattice modulus for the (110) plane $E_{110}^{\prime}$, all plotted in logarithms of the relative values referred at $50^{\circ} \mathrm{C}$. relative values approaches the temperature dependence of $p$, suggesting that the vertical shift factor must be attributed more to the temperature dependence of $E_{\mathrm{cr}}$ than to that of $X_{\mathrm{c}}$.

\section{$\beta$ Dispersion Separated from $E^{*}$}

Figure 8 shows the contribution of the $\beta$ dispersion (primary dispersion) separated by subtracting the contribution of the $\alpha$ dispersion (grainboundary dispersion) in Figure 7 from $E^{*}$ in Figure 5. As can be seen in Figure 8, it is necessary to shift the separated data not only horizontally but also vertically for constructing the master curves of $q E_{\mathrm{am}}^{\prime}$ and $q E_{\mathrm{am}}^{\prime \prime}$. Assuming the tempera-

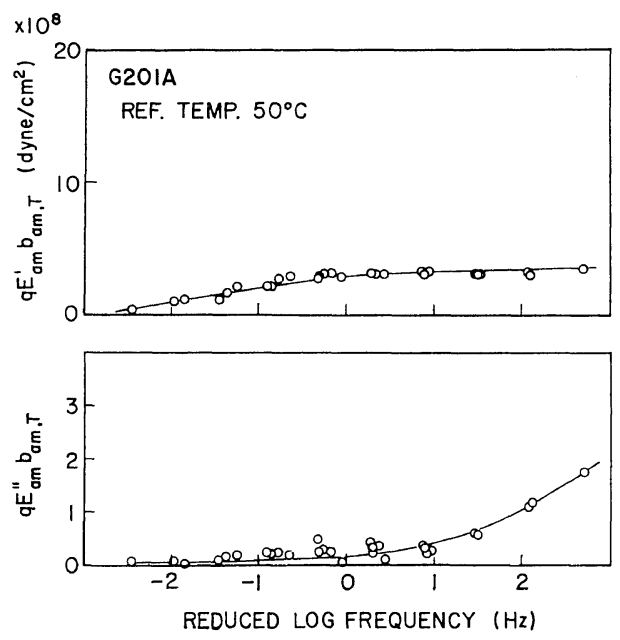

Figure 10. Master curves of the $\beta$ dispersion constructed from $q E_{a m}^{*}$ in Figure 8 .

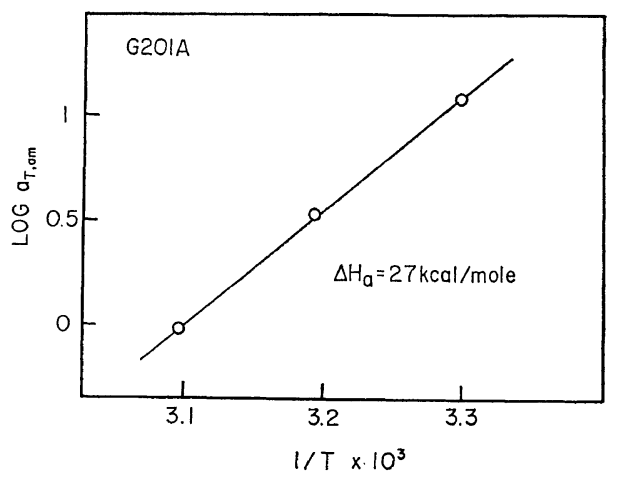

Figure 11. Temperature dependence of the horizontal shift factor required in constructing the master curves of $q E_{\mathrm{am}}^{\prime}$ and $q E_{\mathrm{am}}^{\prime \prime}$. 


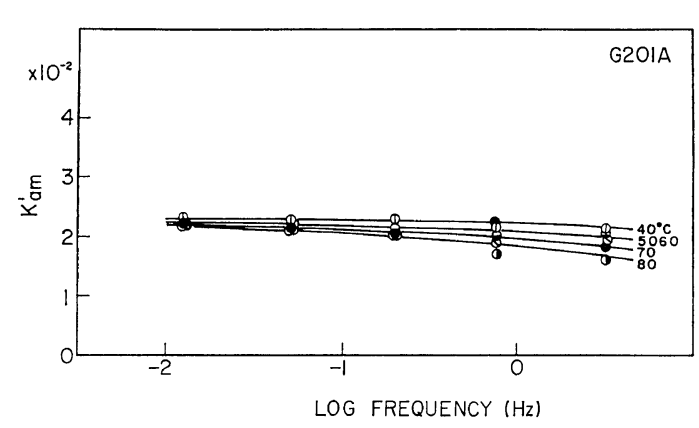

Figure 12. Temperature dependence of the dynamic strain-optical coefficient of the noncrystalline phase, $K_{\mathrm{am}}^{\prime}$, separated from the data of $K^{*}$ on the basis of eq 21.

ture dependence of the vertical shift factor to be similar to that given by eq 30 , the master curves are constructed, as shown in Figure 10, again by a trial and error method under the limited condition that the vertical as well as the horizontal shift factors be identical, between $q E_{\mathrm{am}}^{\prime}$ and $q E_{\mathrm{am}}^{\prime \prime}$. The temperature dependence of the horizontal shift factor thus determined, is found to follow a type of Arrhenius plot, as shown in Figure 11, with the activation energy of the relaxation process being about $27 \mathrm{kcal} / \mathrm{mol}(113 \mathrm{~kJ}$ $\mathrm{mol}^{-1}$ ), while that of the vertical shift factor is found in terms of the value of $b_{0}$ in eq 30 as $0.0174 \mathrm{~K}^{-1}$.

The temperature dependence of $q$, defined by eq 25 , seems small, because $\phi$ and $\nu$ must be primarily small in their temperature dependence. Therefore, the temperature dependence of the vertical shift factor must be ascribed mainly to the temperature dependence of $E_{\mathrm{am}}^{\prime}$ and $E_{\mathrm{am}}^{\prime \prime}$ themselves. The separated data are only a part of the primary dispersion, and may not be sufficient enough to make further discussion about the temperature dependences of $E_{\mathrm{am}}^{\prime}$ and $E_{\mathrm{am}}^{\prime \prime}$.

\section{Significance of Vertical Shift Factor for Optical Data}

The significance of the vertical shift factor for optical data has also been fully discussed in previous papers, ${ }^{9,12}$ in which the strain-optical coefficient is represented in terms of eq 22 and the crystalline and noncrystalline contributions, $K_{\text {cr }}^{*}$ and $K_{\mathrm{am}}^{*}$ are successfully separated from $K^{*}$ for this particular specimen of G201(A). For the separation, the fractional volume of $X_{\mathrm{c}}\left(=1-X_{\mathrm{am}}\right)$ determined from X-ray diffraction measurement, was adopted. Comparing eq 22, in turn, with eq 21 for the present model, the following relation must be validated,

$$
\phi \nu \mu \simeq X_{\mathrm{c}}
$$

and be explained more in terms of the smaller value of 'X-ray crystallinity' $X_{\mathrm{c}}$ than by 'mechanical crystallinity' $\nu \mu$.

As recognized from the previous papers, the separated noncrystalline contribution $K_{\mathrm{am}}^{\prime}$ must be shifted not only horizontally but also vertically for constructing its master curve. The significance of the vertical shift factor may be understood in terms of the temperature dependence of $(1-\phi \nu)$ in eq 21 for the present model as well as that of $K_{\mathrm{am}}^{*}$, if any. Figure 12 shows $K_{\mathrm{am}}^{\prime}$ shifted vertically, by considering the temperature dependence of $(1-\phi \nu)$, where $\phi \nu$ is assumed to be $0.5 X_{c}$ because of the insufficiency of the evaluating method of $\phi \nu$, and $50^{\circ} \mathrm{C}$ is taken as a reference temperature. Considerable, but not fully satisfactory, improvement can be seen.

Finally, in contrast to the mechanical data, it is emphasized that the significance of the vertical shift factor for the optical data must be interpreted mainly in terms of the temperature dependence on the degree of crystallinity.

Acknowledgments. This series of rheo-optical studies on the deformation mechanism of semicrystalline polymers is supported in part by a grant from the Japan-U.S. Cooperative Research Program, Japan Society for Promotion of Science, and the National Science Foundation (U.S.A.), to which the authors are deeply indebted. The authors are also indebted to the Nippon Gosei Kagaku Co. Ltd., Osaka, Japan, and the DaiCell Co. Ltd., Osaka, for financial support through a scientific grant.

\section{REFERENCES}

1. S. Iwayanagi, paper presented at the 2nd Kyoto Seminor on Polymers, Kyoto, 1968.

2. N. Saito, K. Okano, S. Iwayanagi, and T. Hideshima, in "Solid State Physics", H. Ehrenreich, F. Seitz, and D. Turnbull Eds., Vol. 14, p 458, Academic Press, New York, N.Y., 1963.

3. N. G. McCrum and E. L. Morris, Proc. R. Soc. London Ser., A, 292, 506 (1966). 
4. T. Kajiyama, T. Okada, A. Sakoda, and M. Takayanagi, J. Macromol. Sci.-Phys., B7, 583 (1973).

5. T. Kajiyama, T. Okada, and M. Takayanagi, J. Makromol. Sci.-Phys., B9, 35 (1974).

6. M. Takayanagi, J. Makromol. Sci.-Phys., B9, 391 (1974).

7. K. Nagamatsu, Kolloid-Z., 172, 141 (1960).

8. S. Onogi, T. Sato, A. Asada, and Y. Fukui, J. Polym. Sci., Part A-2, 8, 1211 (1970).

9. S. Suehiro, T. Yamada, H. Inagaki, T. Kyu, S. Nomura, and H. Kawai, J. Polym. Sci., Polym. Phys. Ed., in press.
10. S. Suehiro, T. Yamada, H. Inagaki, and $H$. Kawai, Polym. J., 10, 315 (1978).

11. M. Takayanagi, S. Uemura, and S. Minami, J. Polym. Sci., Part C, 5, 113 (1964).

12. T. Kyu, N. Yasuda, S. Suehiro, S. Nomura, and H. Kawai, Polym. J., 8, 565 (1976).

13. K. Miki, K. Hikichi, and M. Kaneko, Jpn. J. Appl. Phys., 6, 931 (1967).

14. K. Miki, J. Yasuda, and M. Kaneko, Jpn. J. Appl. Phys., 8, 159 (1969).

15. Y. Wada and K. Tsuge, Jpn. J. Appl. Phys., 1, 64 (1962).

16. K. Okano, J. Polym. Sci., Part C, 15, 95 (1966). 